\title{
ANÁLISE DO DESEMPENHO DO EDITAL DE 2012 DO PELC ${ }^{1}$
}

Recebido em: 06/02/2017

Aceito em: 21/08/2017

Edmilson Santos dos Santos

Universidade Federal do Vale do São Francisco

Petrolina - PE - Brasil

RESUMO: Como política social, o Programa Esporte e Lazer da Cidade (PELC) se destinam a ofertar políticas de esporte e lazer às regiões mais vulneráveis. Nesse sentido, o presente estudo de caráter descritivo buscou analisar se a aprovação das propostas e implementação garante maior equidade no acesso a essas políticas. A análise dos resultados do Edital de 2012 permitiu verificar que os incentivos à descentralização promovida pelo programa são insuficientes para que ele obtenha efeitos redistributivos e que o número de propostas implementadas compromete os objetivos do PELC.

PALAVRAS CHAVE: Políticas Públicas. Esportes. Atividades de Lazer. Descentralização.

\section{PERFORMANCE ANALYSIS OF THE 2012'S PCSL PUBLIC NOTICE}

ABSTRACT: As a social policy, the Program City's Sport and Leisure (PCSL) aims to offer sports and leisure policies to more vulnerable regions. This way, the present descriptive study seeks to analyze if proposal approval and implementation guarantee more equity on the access to such policies. The analysis of the 2012's public notice allows verifying that incentives to decentralization promoted by the program are insufficient for them to obtain redistributive effect and that the number of implemented proposals compromises PCSL's goals.

KEYWORDS: Public Policies. Sports. Leisure Activities. Decentralization.

\section{Introdução}

A Secretaria de Desenvolvimento de Esporte e Lazer do Ministério do Esporte, em 2003, criou o Programa Esporte e Lazer da Cidade destinado a ofertar atividades de esporte e de lazer. Formulado pelo Governo Federal, sua implementação se dá através

\footnotetext{
${ }^{1}$ Edital Universal CNPq 2014/13.
} 
da descentralização das atividades por meio de convênios com agentes descentralizadores (ou implementadores): governos subnacionais (governos estaduais e prefeituras) e entidades privadas sem fins lucrativos.

O problema que fez surgir o programa está associado a uma avaliação de que há carência de atividades esportivas e de lazer que justificam sua existência. De certa forma, o Perfil dos Municípios Brasileiros, Esporte (2006), produzido pelo Instituto Brasileiros de Geografia e Estatística (IBGE), com base em informação de 2003, confirma essa assertiva. Além de implementar atividades esportivas e de lazer também há a preocupação de que os agentes implementadores se apropriem dessa tecnologia e construam suas próprias políticas públicas. No edital de 2012, está expresso que o programa deve "contribuir com a construção de políticas locais de esporte e de lazer". $\mathrm{Na}$ esteira do que havia sido deliberado na II Conferência Nacional de Esporte sobre a municipalização das políticas públicas de esporte e lazer.

Parece que a premissa básica do PELC é que os governos subnacionais, especialmente os governos municipais, não são ativos na oferta de políticas públicas de esporte e de lazer. Porém, a comparação de gastos entre o Governo Federal e os governos municipais cria um problema para essa tese. O gasto do Governo Federal com o PELC de 2004 a 2011 somou R\$ 238,91 milhões (CASTRO, 2016). No mesmo período, o investimento dos governos municipais na Função Desporto e Lazer (FDL) ${ }^{2}$ foram de 3,34 bilhões de reais. Somente os recursos investidos pelo município de São Paulo no ano de 2011 na FDL foram superiores ao gasto do Ministério do Esporte com o PELC de 2004 a 2011, R\$ 239,52 milhões. Em 2011, 4.989 municípios (89,56\% dos

\footnotetext{
${ }^{2}$ Dados fornecidos pelo Centros de Desenvolvimento de Pesquisas em Políticas de Esporte e Lazer da Rede CEDES Piauí. As informações referentes à FDL foram capturadas junto ao site do Tesouro Nacional (https://siconfi.tesouro.gov.br/siconfi/pages/publi) nos dados referentes às contas anuais no Sistema de Informações Contábeis e Fiscais do Setor Público Brasileiro - Siconfi, entre os dias 10 e 30 de junho de 2016.
} 
municípios brasileiros) fizeram investimentos na FDL. Quando o Edital de 2012 foi lançado já havia, de fato, a municipalização das políticas públicas de esporte e de lazer.

A dotação orçamentária do PELC não é capaz de contribuir, como destaca o programa, para o desenvolvimento de políticas de Estado e nem de efetivar seu objetivo geral: "contribuir com a democratização do acesso ao esporte recreativo e ao lazer, por meio da promoção de ações educativas". Seu desenho denuncia essa limitação já na forma de premiar as propostas.

A forma de implementação do programa é através de núcleos que se diferem em relação à finalidade e ao público alvo. Em 2012, o PELC ofertou três modalidades de núcleos: Núcleo Todas as Idades (NTI); Núcleos para os Povos e Comunidades Tradicionais (NPCT); e Núcleo Vida Saudável (NVS). O NTI se propõe a realizar atividades sistemáticas e assistemáticas com objetivo de democratizar as atividades esportivas e de lazer. Tem como público-alvo todas as faixas etárias e os portadores de deficiência. O NPCT mantem os objetivos do NTI e tem como público-alvo os povos indígenas, os quilombolas, as populações ribeirinhas e as populações rurais. Já o NVS também muda apenas o público-alvo, que são as pessoas acima de 45 anos.

Para as atividades sistemáticas, há cinco faixas de distribuição da quantidade de núcleos: 01 núcleo para cidades com população até 50.000 habitantes; 02, de 50.001 a 100.000 habitantes; 03, de 100.001 a 150.000 habitantes; 04, 150.001 a 200,000 habitantes; 05, para cidades acima de 200.001 habitantes. A limitação do número de núcleos desconsidera as necessidades reais das cidades. Portanto, não se visualiza a universalização nesse modelo.

Os critérios para seleção das propostas servem para excluir proponentes diante, talvez, das restrições orçamentárias. Incapacitados de universalizar o programa, é 
preciso encontrar estratégia para excluir proponentes, aspecto esse pouco explorado pela literatura crítica em educação física. A marca dessa exclusão é a produção de vazio assistencial $^{3}$, grandes áreas do território nacional sem acesso ao PELC.

Quando analisamos o programa sob a égide do federalismo brasileiro, não é possível pensar em sua universalização e consequente democratização dada a autonomia dos governos municipais de conveniarem ou não com o Ministério do Esporte para descentralizarem o programa (ARRETCHE, 2010; MINHOTO, 2014). Não está na autoridade do Governo Federal estabelecer que programas devem ou não ser descentralizados pelas prefeituras. Portanto, o federalismo impõe limite institucional importante à democratização das atividades de esporte e de lazer via programas descentralizados pelo Governo Federal ${ }^{4}$.

No entanto, o papel do Governo Federal na coordenação de políticas que serão descentralizadas está em alcançar o que está proposto no Inciso 3 do Art. 3 da Constituição Federal de 1988 (BRASIL, 1988), a saber, reduzir as desigualdades sociais. Nesse caso, cabe ao Governo Federal coordenar a descentralização do programa de forma a atingir primeiro aquelas regiões onde há carência de políticas sociais (MELO, 2005).

Essa coordenação é feita por meio de incentivos aos agentes descentralizadores de forma que eles sejam induzidos a conveniarem com o Ministério do Esporte. Os incentivos estão associados à oferta de um programa formulado pelo Governo Federal com previsão de pagamento de recursos humanos, da oferta de material de consumo e permanente, de recursos para a formação e para realização de atividades assistemáticas.

\footnotetext{
${ }^{3} \mathrm{O}$ tema do vazio assistencial produzido pelas políticas do Governo Federal foi discutido no trabalho de Santos, Starepravo e Souza Neto (2015).

${ }^{4}$ Para Almeida (2005), no federalismo cooperativo a autonomia decisória é relativa à capacidade de financiamento.
} 
O programa também exige baixa contrapartida financeira (outro incentivo) que pode variar de $2 \%$ do valor pactuado até $20 \%$. No entanto, os incentivos do PELC concorrem com as políticas de esporte das prefeituras, dificultando a cooperação (ARRETCHE; VAZQUEZ; GOMES, 2012; COSTA, 2010).

Como política social, mas sem recursos para universalização do programa, o PELC deve ser capaz de garantir maior equidade no acesso ao esporte e ao lazer. Políticas de equidade precisam ser perseguidas através de instrumentos que permitam a focalização, o tratamento seletivo daqueles em piores condições sociais (PAES; SIQUEIRA, 2008). Ao lançar edital, à luz da política social, cria-se a expectativa de que o programa conseguirá contemplar essa realidade.

Diante desse contexto, o grande desafio do Ministério do Esporte é garantir que o programa alcance de forma prioritária aquelas regiões ou localidades mais vulneráveis. Nesse sentido, o objetivo do estudo foi verificar se a implementação do PELC no ano de 2012 atingiu de forma prioritária os municípios vulneráveis. Para tanto, o presente artigo foi organizado em quatro momentos, fora a introdução: uma breve dissertação sobre a vulnerabilidade; logo após, apresentação do modelo metodológico do estudo e discussão dos resultados. Por último, as considerações finais.

\section{A vulnerabilidade}

Como política social, o programa deve garantir equidade no acesso às atividades esportivas e de lazer. Por não atender a princípios distributivos (universalização), o programa, atendendo a critérios de justiça social, deveria alcançar de forma prioritárias 
aquelas cidades e regiões mais vulneráveis. Parece que o critério "b" da contrapartida do programa tenta estabelecer uma distinção importante do ponto de vista regional de forma a potencializar as prefeituras das regiões Norte, Nordeste e Centro-Oeste. Principalmente as duas primeiras são as regiões que ofertam os menores investimentos no desenvolvimento do esporte (IBGE, 2006).

O vulnerável pode ser alcançado através de duas justificativas distintas. Em termos de justiça social, atender o mais vulnerável responde a necessidade de estabelecer políticas de equidade. Do ponto de vista do modelo de política social (bemestar) de corte liberal, as políticas restritivas de gastos exigem a focalização dos gastos sociais nas populações mais pobres. Nos dois casos, alcançar o vulnerável é tarefa a ser perseguida pelo Estado.

Quando falamos em vulnerável, parece que esse significado é capaz de apontar, no final do processo de significação, um público que está sob-risco social (JANCZURA, 2012). Não são portadores plenos dos direitos sociais garantidos pela Constituição Federal de 1988. A vulnerabilidade pode ser interpretada de forma lato sensu como uma dada realidade social em que determinados grupos, famílias (PETTENGILL; ANGELO, 2005) ou indivíduos estão em condição de desvantagem, marginalidade ou exclusão em relação aos direitos sociais consagrados pelo nosso ordenamento constitucional. A desvantagem, a marginalidade ou a exclusão podem ser espacial (segregação ou prisão), física (eliminação) ou existencial (não possui direitos plenos) (MARANDOLA JR; HOGAN, 2013).

\footnotetext{
5 “4\% (quatro por cento) e $8 \%$ (oito por cento) para Municípios acima de 50.000 (cinquenta mil) habitantes localizados nas áreas prioritárias definidas no âmbito da Política Nacional de Desenvolvimento Regional - PNDR, nas áreas da Superintendência do Desenvolvimento do Nordeste - SUDENE, da Superintendência do Desenvolvimento da Amazônia - SUDAM e da Superintendência do Desenvolvimento do Centro-Oeste - SUDECO". Edital 2012 do PELC. http://www.esporte.gov.br/arquivos/snelis/PELC2012/diretrizesEdital2012V4.pdf
} 
A vulnerabilidade é uma construção social em disputa (BUSSO, 2001). Não há um consenso sobre o que seja ou como pode ser aferida. Porém, há alguns critérios balizadores que nos ajudam a tatear essa realidade. $\mathrm{O}$ critério econômico permite estabelecer, do ponto de vista monetário, um mínimo de recursos que as famílias deveriam dispor para garantir que suas necessidades básicas possam ser contempladas. Do ponto de vista espacial, ser morador de uma determinada região que sofre com o acesso de determinados serviços, como a falta de saneamento básico, por exemplo, fragiliza a saúde da comunidade. O mesmo ocorre com as regiões onde concentram grande parte da violência urbana (CASTRO; ABRAMOVAY, 2002). Portanto, há diferentes formas de identificar a vulnerabilidade.

Dada a complexidade dos fenômenos sociais, a vulnerabilidade não é uma expressão aglutinadora e unificadora de todas as dificuldades por que passam as comunidades e os indivíduos. As vulnerabilidades são múltiplas e o número de exposições a determinados eventos aumenta a fragilidade de determinado grupos e indivíduos (BILAC, 2006).

Alguns critérios (índices sintéticos) têm sido utilizados para estabelecer a distinção, mesmo que frágeis do ponto de vista conceitual, de forma a apontar o grupo que deve receber atenção prioritária por parte do Estado. Entre os indicadores sintéticos mais utilizados e que permite comparações nacionais e internacionais está o Índice de Desenvolvimento Humano (IDH) produzido pelo Programa das Nações Unidas para o Desenvolvimento (PNUD). O IDH contempla três dimensões da vida, saúde, educação e renda, e pode ser classificado em cinco níveis: IDH Muito Baixo (MB), de 0,000 a 0,499; IDH Baixo (B), de 0,500 a 0,599; IDH Médio (M), de 0,600 a 0,699; IDH Alto (A), de 0,700 a 0,799; e IDH Muito Alto (MA), acima de 0,800. 
No Brasil há uma configuração bastante diversa do IDH entre os 5.570 municípios que demarcam com nitidez nossas desigualdades. Como podemos observar na Tabela 1, as regiões Norte e Nordeste não possuem cidades com IDH MA e as regiões Sudeste, Sul e Centro-Oeste não possuem cidades com IDH MB. São as regiões Norte e Nordeste que concentram a maior parte dos municípios vulneráveis do país. No nível de menor vulnerabilidade, a região Sudeste tem o maior percentual de municípios, $65,91 \%(n=29)$.

Tabela 1 - Distribuição dos municípios por região e nível de IDH.

\begin{tabular}{l|c|c|c|c|c|c|c|c|c|c}
\hline \multirow{2}{*}{ Regiões } & \multicolumn{2}{|c|}{$\mathrm{MA}$} & \multicolumn{2}{c|}{$\mathrm{A}$} & \multicolumn{2}{c|}{$\mathrm{M}$} & \multicolumn{3}{c|}{$\mathrm{B}$} & \multicolumn{2}{c}{$\mathrm{MB}$} \\
\cline { 2 - 11 } & $\mathrm{N}^{\mathrm{o}}$ & $\%$ & $\mathrm{~N}^{\mathrm{o}}$ & $\%$ & $\mathrm{~N}^{\mathrm{o}}$ & $\%$ & $\mathrm{~N}^{\mathrm{o}}$ & $\%$ & $\mathrm{~N}^{\mathrm{o}}$ & $\%$ \\
\hline Norte & 0 & 0 & 25 & 1,32 & 226 & 10,12 & 180 & 13,17 & 18 & 56,25 \\
Nordeste & 0 & 0 & 34 & 1,80 & 647 & 28,97 & 1099 & 80,40 & 14 & 43,75 \\
Sudeste & 29 & 65,91 & 871 & 46,13 & 695 & 31,12 & 73 & 5,34 & 0 & 0 \\
Sul & 15 & 34,09 & 768 & 40,68 & 400 & 17,91 & 5 & 0,37 & 0 & 0 \\
Centro-Oeste & 0 & 0 & 190 & 10,06 & 265 & 11,87 & 10 & 0,73 & 0 & 0 \\
\hline
\end{tabular}

Fonte: Relatório PNUD.

A grande maioria dos municípios estão entre os níveis Alto, Médio e Baixo de IDH. No nível Alto, as regiões Sudeste e Sul concentram, juntas, 86,81\%. No nível Médio, as regiões Nordeste e Sudeste têm o maior percentual dos municípios, 59,09\%. No nível Baixo, a maior concentração percentual está na região Nordeste. Em linhas gerais, os municípios das regiões Norte e Nordeste concentram os piores resultados de IDH e as regiões Sudeste e Sul, os melhores.

Do ponto de vista intrarregional, metade dos municípios da região Norte (50,33\%) estão no nível Médio de IDH e outros 40,09\% no nível Baixo, como pode ser observado na (TABELA 2). Com percentuais diferentes, o mesmo acontece com a região Nordeste: 97,32\% dos municípios estão nos níveis Médio e Baixo, com maior concentração no nível Baixo. As regiões Sudeste e Sul têm a maioria de seus municípios 
no nível Alto, 52,22\% e 64,65\%, respectivamente. São as únicas regiões que possuem cidades com nível Muito Alto de IDH. A região Centro-Oeste concentra a maioria de seus municípios no nível Médio de IDH e nenhum município nos dois extremos.

Tabela 2 - Distribuição dos níveis de IDH intrarregiões

\begin{tabular}{|c|c|c|c|c|c|c|c|c|c|c|}
\hline \multirow[t]{2}{*}{ Regiões } & \multicolumn{2}{|c|}{ MA } & \multicolumn{2}{|c|}{$\mathrm{A}$} & \multicolumn{2}{|c|}{$\mathrm{M}$} & \multicolumn{2}{|c|}{$\mathrm{B}$} & \multicolumn{2}{|c|}{$\mathrm{MB}$} \\
\hline & $\mathrm{N}^{\mathrm{o}}$ & $\%$ & $\mathrm{~N}^{\mathrm{o}}$ & $\%$ & $\mathrm{~N}^{\mathrm{o}}$ & $\%$ & $\mathrm{~N}^{\mathrm{o}}$ & $\%$ & $\mathrm{~N}^{\mathrm{o}}$ & $\%$ \\
\hline Norte & 0 & 0 & 25 & 5,57 & 226 & 50,33 & 180 & 40,09 & 18 & 4,01 \\
\hline Nordeste & 0 & 0 & 34 & 1,90 & 647 & 36,06 & 1099 & 61,26 & 14 & 0,78 \\
\hline Sudeste & 29 & 1,74 & 871 & 52,22 & 695 & 41,67 & 73 & 4,38 & 0 & 0 \\
\hline Sul & 15 & 1,26 & 768 & 64,65 & 400 & 33,67 & 5 & 0,42 & 0 & 0 \\
\hline Centro-Oeste & 0 & 0 & 190 & 40,86 & 265 & 56,99 & 10 & 2,15 & 0 & 0 \\
\hline Total & 44 & 0,79 & 1888 & 33,93 & 2233 & 40,13 & 1367 & 24,57 & 32 & 0,58 \\
\hline
\end{tabular}

$\mathrm{O}$ indicador IDH pode ajudar a sinalizar as prioridades de atuação de programas coordenados pelo Governo Federal e descentralizados pelos municípios ${ }^{6}$. Se utilizarmos apenas o critério alta vulnerabilidade alcançado pelo indicador IDH MB, as regiões Norte e Nordeste reúnem os municípios de maior vulnerabilidade no Brasil. Dado o número reduzido de municípios que se encontram nessa situação $(\mathrm{n}=32)$, podemos alargar o critério de forma a incorporar o IDH B $(n=1367)$. Como indica a tabela 1 , mais de $93,71 \%$ estão no Norte e Nordeste.

Tendo em vista a função precípua da União na diminuição das desigualdades regionais, cabe a ela estruturar políticas a serem descentralizadas e fornecer incentivos seletivos de forma a combater as desigualdades de acesso às políticas públicas, principalmente as sociais. Diante desse quadro, torna-se relevante compreender quais os resultados alcançados pelo Edital de 2012 do PELC no sentido de contemplar os municípios com maior vulnerabilidade.

\footnotetext{
${ }^{6}$ Um exemplo desse tipo de política foi o Projeto Alvorada criado pelo Governo Federal em 2001 (MENEZES; SANTOS, 2001).
} 


\section{Metodologia}

O estudo de natureza descritiva teve por objetivo verificar se o Edital 2012 garantiu a descentralização do PELC de forma a atingir os municípios de maior vulnerabilidade nos convênios realizados com as prefeituras. Como objetivo específico, analisamos a distribuição das propostas aprovadas por região e nível de desenvolvimento humano.

O marcador de vulnerabilidade nesse trabalho foi o Índice de Desenvolvimento Humano (IDH). O IDH foi coletado no Atlas do Desenvolvimento Humano no Brasil 2013 do Programa das Nações Unidas para o Desenvolvimento. Apenas 6 cidades não apresentaram $\mathrm{IDH}^{7}$. Os dados referentes ao Programa Esporte e Lazer da Cidade foi fornecido pelo Ministério dos Esportes ${ }^{8}$. Os dados compuseram um banco de dados do programa e foram submetidos a estatística descritiva por meio do programa SPSS versão 17.

Para análise do programa, utilizamos como unidade de análise o município através de convênios realizados com prefeituras, e não foi feita distinção entre as modalidades do PELC (todos os núcleos foram contemplados). A análise empreendida busca identificar três cenários no que concerne a implementação: concentração quando a distribuição percentual do programa privilegia os municípios menos vulneráveis, promovendo uma distorção alocativa negativa; reprodução - a distribuição percentual do programa reproduz o status quo em termos de vulnerabilidade, considerando uma margem de erro de 2 pontos percentuais para cima e para baixo; redistribuição - quando a distribuição percentual do programa privilegia os municípios

\footnotetext{
${ }^{7}$ Uma cidade da região Norte, 4 da região Sul e 1 da região Centro-Oeste.

${ }^{8}$ Relatório enviado no dia 2/5/2016 apontando os municípios contemplados em 2012. Protocolo Lei de Acesso à Informação: 58750000093201645.
} 
mais vulneráveis, promovendo uma distorção alocativa positiva. Duas dimensões foram analisadas: a primeira, verificar a participação dos municípios que tiveram suas propostas aprovadas; a segunda, os municípios contemplados.

\section{Análise dos Resultados}

Dois aspectos iniciais chamam atenção. Primeiro, o baixo número de propostas elaboradas pelas prefeituras que foram aprovadas no Edital de 2012, 262 (4,7\%). Menos de $5 \%$ das prefeituras tiveram suas propostas aprovadas, resultado incompatível com a democratização da política. Segundo, das propostas aprovadas apenas $26(0,46 \%)$ foram implementadas. De um modo geral esse resultado descaracteriza o programa.

A distribuição das propostas aprovadas por região Tabela 3 também revela informações importantes do programa. A distribuição das propostas aprovadas garantiu que $98,02 \%(n=99)$ das que se encontram nos níveis de maior vulnerabilidade fossem das regiões que concentram o maior número de municípios vulneráveis (Norte e Nordeste). Esse resultado indica que a procura pelo programa tem recebido atenção dos municípios que mais precisam de políticas sociais (corresponde a 38,54\% das propostas). Parece não haver lacuna informacional ${ }^{9}$.

Comparando os dois extremos da escala de vulnerabilidade (IDH A e MA x B e MB) pudemos constatar que os municípios mais vulneráveis com propostas aprovadas representam $38,54 \%$ do total. Para os municípios menos vulneráveis esse valor ficou em 22,9\%. Os municípios mais vulneráveis obtiveram maior eficiência na aprovação das propostas, comparativamente aos menos vulneráveis.

\footnotetext{
9 Déficit de informação é uma das variáveis que pode comprometer a descentralização das políticas (MACHADO, 2008).
} 
Tabela 3 - Distribuição das propostas aprovadas entre as regiões e níveis de IDH

\begin{tabular}{|c|c|c|c|c|c|c|c|c|c|c|}
\hline \multirow[t]{2}{*}{ Regiões } & \multicolumn{2}{|c|}{ MA } & \multicolumn{2}{|c|}{ A } & \multicolumn{2}{|c|}{$M$} & \multicolumn{2}{|c|}{ B } & \multicolumn{2}{|c|}{ MB } \\
\hline & $\mathrm{N}^{\mathrm{o}}$ & $\%$ & $\mathrm{~N}^{\mathrm{o}}$ & $\%$ & $\mathrm{~N}^{\mathrm{o}}$ & $\%$ & $\mathrm{~N}^{\mathrm{o}}$ & $\%$ & $\mathrm{~N}^{\mathrm{o}}$ & $\%$ \\
\hline Norte & 0 & 0 & 0 & 0 & 2 & 1,98 & 2 & 2 & 0 & 0 \\
\hline Nordeste & 0 & 0 & 2 & 3,51 & 61 & 60,40 & 96 & 96 & 1 & 100 \\
\hline Sudeste & 2 & 66,66 & 23 & 40,35 & 15 & 14,85 & 1 & 1 & 0 & 0 \\
\hline Sul & 1 & 33,33 & 20 & 35,09 & 9 & 8,91 & 1 & 1 & 0 & 0 \\
\hline Centro-Oeste & 0 & 0 & 12 & 21,05 & 14 & 13,86 & 0 & 0 & 0 & 0 \\
\hline
\end{tabular}

Fonte: Dados da pesquisa.

Considerando as propostas aprovadas como o universo das ações a serem implementadas e uma margem de erro de $2 \%$ para cima ou para baixo (comparando distribuição percentual das Tabelas 1 e 3), é possível constar na distribuição horizontal das propostas (por IDH) que:

a) Em três cenários houve ganho de representação da participação dos municípios da região Nordeste e elas foram em direção aos municípios mais vulneráveis. Em dois níveis de IDH, M e MB, o ganho foi expressivo (28,97\% para 60,4\% - M; 43,75\% para 100\% - MB). No nível de IDH B, também houve um ganho. Isso significa que os municípios vulneráveis da região Nordeste estavam melhor capacitados para apresentar propostas qualificadas. No IDH A manteve-se a mesma representação, considerando a margem de erro, $(3,51 \%$ para $1,8 \%)$.

b) A região Norte perdeu representação em todos os níveis de IDH. A pior perda se deu no cenário mais vulnerável, IDH MB. Ele é responsável pela distribuição de $56,25 \%$ dos municípios nesse cenário e não conseguiu contemplar nenhuma proposta.

c) As regiões mais ricas, Sudeste e Sul, não obtiveram ganho em nenhum nível de IDH.

d) A região Centro-Oeste conseguiu obter ganho no nível de IDH A $(10,06 \%$ para $21,05 \%)$. 
Em termos de propostas aprovadas, a região Nordeste foi a maior beneficiária do processo de seleção. O resultado da região Nordeste não permite aludir que os municípios vulneráveis das regiões com maior percentual de municípios vulneráveis foram privilegiados. Outro fato importante é que os municípios das regiões mais desenvolvidas, em nenhum cenário, ampliaram sua participação percentual.

Tabela 4 - Distribuição intrarregião das propostas aprovadas por níveis de IDH

\begin{tabular}{|c|c|c|c|c|c|c|c|c|c|c|}
\hline \multirow[t]{2}{*}{ Regiões } & \multicolumn{2}{|c|}{ MA } & \multicolumn{2}{|c|}{ A } & \multicolumn{2}{|c|}{$\mathrm{M}$} & \multicolumn{2}{|c|}{ B } & \multicolumn{2}{|c|}{ MB } \\
\hline & $\mathrm{N}^{\mathrm{o}}$ & $\%$ & $\mathrm{~N}^{\mathrm{o}}$ & $\%$ & $\mathrm{~N}^{\mathrm{o}}$ & $\%$ & $\mathrm{~N}^{\mathrm{o}}$ & $\%$ & $\mathrm{~N}^{\mathrm{o}}$ & $\%$ \\
\hline Norte & 0 & & 0 & & 2 & 50 & 2 & 50 & 0 & 0 \\
\hline Nordeste & 0 & & 2 & 1,25 & 61 & 38,12 & 96 & 60 & 1 & 0,6 \\
\hline Sudeste & 2 & 4,87 & 23 & 50,09 & 15 & 36,58 & 1 & 2,43 & 0 & \\
\hline Sul & 1 & 3,22 & 20 & 64,51 & 9 & 29,03 & 1 & 3,22 & 0 & \\
\hline Centro-Oeste & 0 & & 12 & 46,15 & 14 & 53,85 & 0 & & 0 & \\
\hline Total & 3 & 1,14 & 57 & 21,76 & 101 & 38,55 & 100 & 38,17 & 1 & 0,38 \\
\hline
\end{tabular}

Fonte: Dados da pesquisa.

A distribuição das propostas intrarregião apresenta os seguintes resultados (TABELA 4):

a) A região Norte obteve resultado redistributivo ao promover distribuição alocativa positiva no IDH B (passou de 40,09\% para 50\%) com a perda de representação daqueles de IDH MB e A. Os municípios mais vulneráveis $(n=18)$ não conseguiram enviar ou ter suas propostas contempladas.

b) A região Nordeste manteve o status quo. As propostas aprovadas não melhoram a posição dos municípios mais vulneráveis.

c) A região Sudeste melhorou a participação percentual dos municípios menos vulneráveis, IDH MA, (1,74\% para 4,87\%), promovendo distribuição alocativa negativa.

d) A região Sul conseguiu distribuição alocativa positiva em direção a sua região mais vulnerável $(0,42 \%$ para $3,22 \%)$ (efeito redistributivo). 
e) A região Centro-Oeste obteve distorção alocativa negativa ao melhorar a posição dos municípios menos vulneráveis do IDH A, em detrimento dos municípios do IDH B e M (efeito concentrador).

Internamente às regiões, observa-se a melhora na representação dos municípios de IDH B apenas das regiões Norte e Sul, enquanto a distribuição alocativa negativa se deu em dois cenários, Sudeste (MA) e Centro Oeste (A). No âmbito geral, houve ganho alocativo positivo na região de IDH B (24,57\% para $38,17 \%)$ enquanto a distorção alocativa negativa se deu no IDH A $(0,79 \%$ para $1,14 \%)$.

Os resultados que aparecem na Tabela 5 comprometem e inviabilizam as duas análises aqui pretendidas. O Edital de 2012 do PELC implementou apenas 26 propostas. Isso significa $10 \%$ das propostas aprovadas e $0,46 \%$ dos municípios brasileiros. $\mathrm{O}$ número de municípios vulneráveis contemplados $\left(n^{\circ} 4\right)$ foi menor que dos municípios contemplados de IDH A (nº 12), promovendo concentração de recursos nos municípios menos vulneráveis. Ou seja, os critérios de seleção das propostas a serem contempladas privilegiaram os municípios menos vulneráveis em detrimento dos mais vulneráveis. Com isso, a tão esperada justiça social, no âmbito do PELC, é descartada, dispensada. Outro aspecto que depõe contra o programa foi o fato da região Norte, com elevado número de municípios vulneráveis, não conseguir implementar nenhuma proposta.

Tabela 5 - Propostas implementadas no Edital de 2012 do PELC por região e nível de IDH.

\begin{tabular}{l|c|c|c|c|c}
\hline Região & MA & A & M & B & MB \\
\hline Norte & 0 & 0 & 0 & 0 & 0 \\
Nordeste & 0 & 0 & 7 & 4 & 0 \\
Sudeste & 0 & 5 & 3 & 0 & 0 \\
Sul & 0 & 5 & 0 & 0 & 0 \\
Centro-Oeste & 0 & 2 & 0 & 0 & 0 \\
\hline
\end{tabular}

Fonte: Dados da pesquisa. 
Para o critério de vulnerabilidade estabelecida no estudo, é negativo o fato de que apenas $4 \%(n=4)$ das propostas oriundas dos municípios de maior vulnerabilidade (IDH B e MB) tenham sido implementadas. Percentual esse muito menor que a taxa de sucesso na implementação dos municípios de menor vulnerabilidade (IDH A) que foi de $21,05 \%$ (n 8).

Diante das carências de políticas públicas de esporte e lazer que justificam a existência do programa, os resultados alcançados no ano de 2012 sinalizam que há problemas em seu desenho. A forma como a política está construída ignora a desigualdade de acesso que o fez surgir. Mesmo que a literatura sobre descentralização de políticas sociais suporte a ideia de que não necessariamente haverá diminuição de assimetrias (MACHADO, 2008; ARRETCHE; MARQUEZ, 2007; ARRETCHE; VAZQUEZ; GOMES, 2012; SOUZA, 2001), o resultado aponta para limitações severas do programa de atuar como política social com base no Inciso III do Art. 3 da CF/88.

Talvez o cálculo do custo-benefício do programa (BONFIM; SILVA, 2003) não seja atraente para mobilizar a máquina pública das prefeituras. Também é ponto pacífico na literatura sobre descentralização de políticas que a distribuição dos recursos é afetada por fatores institucionais (como os incentivos à descentralização e a forma de contemplação das propostas). No Edital do PELC/2012 não há mecanismos que promovam diferenciadamente aquelas cidades mais vulneráveis. Nesse caso, há limitações para o Governo Federal coordenar a política (MENDES, 1989) de forma a garantir resultados redistributivos. 


\section{Conclusão}

Qualquer observação conclusiva sobre as distorções alocativas do PELC no ano de 2012 se fragilizam diante do pequeno número de municípios $(0,46 \%$ do total $)$ que conveniaram com o programa. $\mathrm{O}$ fato de ter aprovado apenas 4 propostas de municípios de maior vulnerabilidade deveria ascender um alerta ao programa. As propostas rejeitadas, 96\% no IDH B contra 78,95\% no IDH A, são um forte indicativo de que o desenho do programa atua de forma contrária às políticas sociais.

Se propostas não foram encaminhadas, há algum problema na divulgação ou desenho do programa que não apresenta incentivos suficientes (ou atraentes) aos municípios. No entanto, priorizar os menos vulneráveis é uma opção política que contraria as finalidades do programa. Esse resultado indica que o programa deveria rever seu desenho e seus incentivos à descentralização.

\section{REFERÊNCIAS}

ALMEIDA, M. H. T. Recentralizando a federação? Revista Sociologia Politica. Curitiba, 24, p. 29-40. Jun, 2005.

ARRETCHE, M. Federalismo e igualdade territorial: uma contradição em termos? Dados, Rio de Janeiro, v. 53, n. 3, p. 587-620, 2010.

.; VAZQUEZ, D.; GOMES, S. As relações verticais na federação: explorando o problema da descentralização e da autonomia. Democracia, federalismo e centralização no Brasil, p. 145-173, 2012.

ARRETCHE, M., VAZQUEZ, D., GOMES, S. Descentralização e autonomia: deslocando os termos do debate. In. LAVALLE, A. G. (Org.) O Horizonte da política - Questões emergentes e agendas de pesquisa. São Paulo: UNESP, 2012.

; MARQUES, E. Condicionantes locais da descentralização das políticas de saúde. In: HOCHMAN, G.; ARRETCHE, M.; MARQUES, E. (Org.) Políticas públicas no Brasil. Rio de Janeiro: Ed. FIOCRUZ, 2007. p. 173-204.

BILAC, Elisabete Dória. Gênero, vulnerabilidade das famílias e capital social: algumas reflexões. Novas metrópoles paulistas. População, vulnerabilidade e segregação. Campinas: Unicamp/Nepo, 2006. p. 51-65. 
BONFIM, W. L. S., SILVA, I. N. Instituições políticas, cidadania e participação: a mudança social ainda é possível? Rev. Sociol. Polít., Curitiba. 21. p. 109-123, nov, 2003.

BRASIL. Constituição da República Federativa do Brasil (1988). Diário Oficial [da] República Federativa do Brasil, Brasília, DF, 05 out. 1988.

BUSSO, G. Vulnerabilidad Social: nociones e implicancias de políticas para América Latina y el Caribe a comienzos del Siglo XXI. SEMINARIO INTERNACIONAL SOBRE LAS DIFERENTES EXPRESIONES DE LA VULNERABILIDAD SOCIAL EN AMÉRICA LATINA Y EL CARIBE. CEPAL/CELADE. Anais...Santiago de Chile, 2001.

CASTRO, S. B. E. Políticas públicas para o esporte e lazer e o ciclo orçamentário brasileiro (2004-2011): prioridades e distribuição de recursos durante os processos de elaboração e execução orçamentária. Universidade Federal do Paraná, Programa de PósGraduação em Educação Física, 2016. p. 382.

CASTRO, Mary Garcia; ABRAMOVAY, Miriam. Jovens em situação de pobreza, vulnerabilidades sociais e violências. Cadernos de pesquisa, n. 116, p. 143-176, 2002.

COSTA, V. M. F. C. Federalismo e relações intergovernamentais: implicações para a reforma da educação no Brasil. Educação e Sociedade, Campinas, v. 13, n. 112, p. 729-748, jul./set. 2010.

IBGE. Perfil dos municípios brasileiros: esporte 2003. Rio de Janeiro: IBGE, 2006.

JANCZURA, Rosane. Risco ou vulnerabilidade social? Textos \& Contextos, v. 11, n. 2, p. 301-308, 2012.

MACHADO, José Angelo. Gestão de políticas públicas no Estado federativo: apostas e armadilhas. Dados-Revista de Ciências Sociais, p. 433-457, 2008.

MARANDOLA JR, Eduardo; HOGAN, Daniel Joseph. Vulnerabilidades e riscos: entre geografia e demografia. Revista Brasileira de Estudos de População, v. 22, n. 1, p. 29-53, 2013.

MELO, M. A. O sucesso inesperado das Reformas de Segunda Geração: federalismo, reformas constitucionais e política social. Dados, Rio de Janeiro, v. 48, n. 4, p. 845-889, 2005.

MENDES, Constantino Cronemberger. O território e o arranjo federativo para o desenvolvimento brasileiro: o caso do nordeste. Federalismo à Brasileira questões para discussão, 1989.

MENEZES, Ebenezer Takuno de; SANTOS, Thais Helena dos. Verbete Projeto Alvorada. Dicionário Interativo da Educação Brasileira - Educabrasil. São Paulo: Midiamix, 2001. Disponível em: http:/www.educabrasil.com.br/projeto-alvorada/ . Acesso em: 01 fev. 2017. 
MINHOTO, A. C. B. Federalismo, estado federalista e a revalorização do município: um novo caminho para o século XXI? Revista Brasileira de Políticas Públicas, v. 3, n. 2, p. 52-64, 2014.

PAES, N. L.; SIQUEIRA, M. L. Desenvolvimento regional e federalismo fiscal no Brasil: em busca da igualdade na distribuição de receitas. Economia Aplicada, São Paulo, v. 12, n. 4, p. 707-742, out./dez. 2008.

PETTENGILL, Myriam Aparecida Mandetta; ANGELO, Margareth. Vulnerabilidade da família: desenvolvimento do conceito. Revista Latino-Americana de Enfermagem, 2005 .

SANTOS, E. S.; STAREPRAVO, F. A; SOUZA NETO, M. S. Programa Segundo Tempo e o vazio assistencial na região Nordeste. Movimento, Porto Alegre, v. 21, p. 759-771, 2015.

SOUZA, Celina. Federalismo e gasto social no Brasil: tensões e tendências. Lua Nova, São Paulo, v. 52, p. 5-28, 2001.

\section{Endereço do Autor:}

Edmilson Santos dos Santos

Av. Tancredo Neves 80/302 - Centro

Petrolina - PE - 56.304-190

Endereço Eletrônico: edmilson.santos@univasf.edu.br 